\title{
Compatibilidad entre nueve cepas de Biomphalaria glabrata de áreas endémicas y no endémicas y una cepa deSchistosoma mansoni venezolanas
}

\author{
Compatibility between nine strains of Biomphalaria glabratacoming from endemic \\ and non-endemic areas and one strain of Schistosoma mansonifrom Venezuela
}

Luz A. Pino ${ }^{1}$, Liboria Matinella² y Gustavo Morales C. ${ }^{1}$

\begin{abstract}
Resumen Se infectaron experimentalmente 9 lotes de 32 caracoles B. glabrata (de 5 a $7 \mathrm{~mm}$ de diámetro) con miracidios de la cepa C5 de Schistosoma mansoni a razón de 5 miracidios por caracol, pertenecientes a las siguientes cepas: En el área endémica de transmisión de Esquistosomiasis mansoni a) Sector Puerta Negra, Lago Valencia, b) Cagua c) Ingenio Bolívar (Estado Aragua) d) Mariara e) Caserío El 25 f) Güigüe (Estado Carabobo). Fuera del area endémica de transmisión g) Anzoátegui (Estado Lara), h) Chabasquén (Estado Portuguesa), i) Sector La Elvira, Caripe (Estado Monagas). El período prepatente intramolusco, osciló entre 23 y 25 días, para las 9 cepas evaluadas. La duración total de la infección fue muy variable desde 20 días para la cepa Chabasquén, hasta 93 días para la de Güigüe.La producción total promedio de cercarias al tercer día de iniciada la emisión varió desde $X=74,4$ para la cepa de Mariara, hasta $X=591,7$ para la cepa de Chabasquén. Se evidenció la existencia de diferencias estadísticamente significativas $(H=97,4, P<0,05)$ en la producción total de cercarias al tercer día de iniciada la emisión, detectándose diferencias estadísticamente significativas para casi todas las 36 combinaciones, excepto para las cuatro siguientes: Mariara/Ingenio Bolívar, Cagua/Caserío El 25, Lago de Valencia/Güigüe y Güigüe/Caripe.En lo que respecta al porcentaje de caracoles que presentaron cura espontánea, los valores mas elevados se obtuvieron en las cepas del Lago de Valencia (88,8\%), Cagua (85,2\%), Chabasquén (82,6\%), Caripe $(82,6 \%)$ y Anzoátegui (80\%). Mientras que el porcentaje mas bajo se obtuvo para la cepa de Güigüe $(21,4 \%)$.
\end{abstract}

Palabras-claves: Schistosoma mansoni. Biomphalaria glabrata. Compatibilidad genética. Cepas. Venezuela.

\begin{abstract}
Nine lots of $32 \mathrm{~B}$. glabrata snails $(5-7 \mathrm{~mm}$ in diameter) from the following strains from the endemic area: Cagua, Valencia Lake (Puerta Negra Sector), Ingenio Bolívar (Aragua State), Mariara, Caserío El 25 and Güigüe (Carabobo state), and from the non-endemic area: Anzoátegui (Lara state), Chabasquén (Portuguesa state) and Caripe (Monagas state) were experimentally infected (5 miracidiums/snail) with C5 Schistosoma mansoni strain. The intramolluscal prepatent period oscillated between 23 and 25 days for the nine strains. The duration of intramolluscal infection varied a lot, from 20 days in the case of the Chabasquén strain to 93 days for the Güigüe strain. The average cercariae production in the 3rd day of emission varied from $X=74.4$ for the Mariara strain to 591.7 in the case of the Chabasquén strain. Significant statistical differences were found in relation to the total number of emitted cercariae in the 3rd day $(H=97.4 ; P<0.05)$, with significant statistical differences detected among most of the 36 strain combinations, except for the following: Mariara/Ingenio Bolívar, Cagua/ Cas. El 25, Valencia Lake/Güigüe and Güigüe/Caripe. The strains of Valencia Lake (88.8\%), Cagua (85.2), Chabasquén (82.6\%) and Caripe (82.6\%) showed the highest rates of self-cure, whereas the Güigüe strain showed the lowest: $21.4 \%$.
\end{abstract}

Key-words: Schistosoma mansoni. Biomphalaria glabrata. Genetic compatibility. Strains. Venezuela.

\footnotetext{
1. Laboratorio de Parasitología, Instituto de Investigaciones Veterinarias, Centro Nacional de Investigaciones Agropecuarias; 2. Laboratorio Malacológico, Dpto. de Parasitosis intestinales y Esquistosomiasis, Dirección de Endemias Rurales, M.S.A.S., Maracay, Edo. Aragua, Venezuela. Dirección para correspondencia: Dra. L.A. Pino de Morales. Lab. de Parasitología, Instituto de Investigaciones Veterinarias/CENIAP/ FONAIAP. Av. Las Delicias, Maracay, Estado Aragua, Venezuela.

Fax: 001603 514-1296

e-mail: gamc@cantv.nt.ve; luz_arelis@hotmail.com

Recebido para publicação em $24 / 8 / 98$.
} 
En Venezuela el área endémica bilharzígena es relativamente pequeña, tiene una extensión de aproximadamente $15.000 \mathrm{~km}^{2}$. En esta zona habita una tercera parte de los habitantes del país ${ }^{1}$. Debido a lo restringido de la zona, el Ministerio de Sanidad y Asistencia Social se había trazado como meta, lograr la erradicación de la esquistosomiasis en la década de los 80 .

La prevalencia de casos en 1996 llegó a descender a 1,39\%. Sin embargo, recientemente se reporta la reinfección con Biomphalaria glabrata (Say, 1818) de algunos cursos de agua y el aumento de la prevalencia de Schistosoma mansoni Sambon, 1907 en algunas áreas².

En Venezuela la esquistosomiasis se considera una enfermedad moderada con pocas manifestaciones clínicas y bajas cargas parasitarias, en donde el $80 \%$ de los infectados elimina menos de 100 huevos por gramo de heces, lo cual debido a la baja sensibilidad de las pruebas parasitológicas hace que se subestime la verdadera prevalencia de la enfermedad y que lejos de lograr su erradicación la transmisión continúe ocurriendo. Actualmente existe la posibilidad de que se incremente el riesgo de transmisión debido a la adaptación del caracol hospedador intermediario a áreas suburbanas y a zonas situadas fuera del área endémica tradicional en estados como Monagas, Lara, Cojedes y Portuguesa.

A pesar de que es bien conocida la distribución geográfica de las poblaciones de caracoles que forman las diferentes cepas de B. glabrata en Venezuela, las relaciones de compatibilidad entre dichas cepas y las de S. mansoni parecen no estar aún bien definidas. Este conocimiento es de gran importancia para establecer el riesgo potencial de instalación de un foco esquistosomiásico en zonas hasta ahora consideradas no endémicas y además porque se ha establecido una relación directa entre la susceptibilidad de los caracoles $B$. glabrata y la patogenia de $S$. mansoni a nivel del hospedador definitivo ${ }^{17}{ }^{18}$, asi como variaciones en la sensibilidad del parásito ante las drogas.

\section{MATERIAL Y METODOS}

Para infectar los caracoles de las 9 cepas de B. glabrata, se utilizaron miracidios de la Cepa C5 de S. mansoni. Esta cepa fué aislada en el Laboratorio Malacológico de la Dirección de Malariología y Saneamiento Ambiental, M.S.A.S. (Maracay, Edo. Aragua, Venezuela) a partir de los huevos presentes en las heces de un paciente de la ciudad de San Mateo, Edo. Aragua y es mantenida en pasajes sucesivos por caracoles $B$. glabrata, cepa Cagua, Edo. Aragua, desde 1989. Para las infecciones experimentales se utilizaron 5 miracidios por caracol.

Se utilizó un total de 288 especímenes de B. glabrata de 5 a $7 \mathrm{~mm}$ de diámetro. Se conformaron 9 lotes, a razón de 32 caracoles/lote, de las cepas siguientes:

Area endémica de transmisión de esquistosomiasis mansoni: Sector Puerta Negra, en el Lago de Valencia; Cagua; Ingenio Bolívar (Estado Aragua) Mariara; Caserío El 25; Güigüe (Estado Carabobo)

Fuera del área endémica de transmisión:

Anzoátegui (Estado Lara); Chabasquén (Estado Portuguesa);

Sector La Elvira, en Caripe (Estado Monagas).
Todos los caracoles, pertenecientes a las distintas cepas evaluadas, fueron mantenidos en idénticas condiciones de laboratorio: en ausencia de luz, a una temperatura de 25 a $26^{\circ} \mathrm{C}$; el pH del agua se mantuvo entre 7,4 y 8 y la concentración de Carbonato de Calcio aproximadamente igual a $18 \mathrm{ppm}$. El agua utilizada en los acuarios era declorinada y dentro de los mismos se utilizaron plantas acuáticas del género Elodea. La alimentación de los caracoles fue a base de lechuga fresca.

Se calcularon las estadísticas descriptivas para el número total de cercarias emitidas al tercer día de iniciada la emisión.

En vista de que la distribución de los datos del número de cercarias emitidas al tercer día no se ajustó a una distribución normal, como lo demuestra el hecho de que en todos los casos la varianza fue muy superior a la media, se recurrió al empleo del ANOVAno paramétrico de Kruskal-Wallis, para comparar las 9 cepas de $B$. glabrata en relación al mencionado criterio. En vista de que se encontraron diferencias estadísticamente significativas entre las cepas, se aplicó como prueba a posteriori, la mínima diferencia significativa ${ }^{11}$. 


\section{RESULTADOS}

La duración del período prepatente intramolusco, es decir aquel comprendido entre el día de la infección miracidiana de los caracoles y el día de la primera emisión de cercarias, fue bastante homogénea, oscilando entre 23 y 25 días, para las 9 cepas evaluadas de B. glabrata (Tabla 1).

En la duración de la infección, es decir el total de días de emisión ceracariana, si se observó gran variabilidad, desde un mínimo de 20 días para la cepa de Chabasquén y 22 para la de Caripe, hasta un máximo de 93 días para la cepa de Güigüe, seguida por Caserío El 25 con 64 días e Ingenio Bolívar con 47 días (Tabla 1).
Otro aspecto en el cual se observó gran variabilidad fue en la producción total de cercarias al tercer día de iniciada la emisión, la cual se extendió desde 1934 cercarias para la cepa de Mariara hasta un máximo de 13608 en la cepa de Chabasquén, seguida por la de Cagua (11852) y Caserío El 25 (11567) (Tabla 1).

En cuanto al porcentaje de caracoles que presentaron cura espontánea, es decir los caracoles sobrevivientes que dejaron de emitir y quedan en capacidad de reinfectarse ${ }^{35}$ el mayor porcentaje le correspondió a la cepa del Lago de Valencia $(88,8 \%)$, seguido por Cagua $(85,2 \%)$,

Tabla 1 - Tasa de infección (T.I.), duración del período pre-patente intramolusco (PPIM) y de la infección (DI), número de cercarias producidas al tercer día de iniciada la emisión (NCP), porcentaje de B. glabrata que presentó cura espontánea (NCE), media aritmética $(X)$ y varianza $(s+)$ de la producción de cercarias al tercer día de iniciada la emisión, para las nueve cepas de B. glabrata infectadas experimentalmente.

\begin{tabular}{lccccccc}
\hline $\begin{array}{l}\text { Cepa de } \\
\text { B. glabrata }\end{array}$ & T.I.(\%) & $\begin{array}{c}\text { PPIM } \\
\text { (días) }\end{array}$ & $\begin{array}{c}\text { D.I } \\
\text { (día) }\end{array}$ & NCP & $\begin{array}{c}\text { NCE } \\
\text { (\%) }\end{array}$ & X & s $^{2}$ \\
\hline Lago de Valencia & 84,4 & 25 & 31 & 8868 & 88,8 & 328,4 & 29584 \\
Mariara & 81,3 & 23 & 26 & 1934 & 53,8 & 74,4 & 7396 \\
Anzoátegui & 62,5 & 25 & 32 & 10566 & 80,0 & 528,3 & 97781,3 \\
Ingenio Bolívar & 65,6 & 25 & 47 & 2693 & 61,9 & 128,2 & 7885,4 \\
Cagua & 84,4 & 25 & 35 & 11852 & 85,2 & 438,9 & 46354,1 \\
Caserío El 25 & 78,1 & 25 & 64 & 11567 & 64,0 & 462,7 & 69063,8 \\
Chabasquén & 71,9 & 24 & 20 & 13608 & 82,6 & 591,7 & 95048,9 \\
Caripe & 71,9 & 24 & 22 & 5058 & 82,6 & 219,9 & 26830,4 \\
Güigüe & 87,5 & 25 & 93 & 7556 & 21,4 & 269,9 & 23409,0 \\
\hline
\end{tabular}

Chabasquén (82,6\%) y Caripe (82,6\%); el menor porcentaje se observó en la cepa Güigüe $(21,6 \%)$ (Tabla 1).

Las estadísticas descriptivas del número total de cercarias emitidas al tercer día de iniciada la emisión, para las 9 cepas de B. glabrata pueden ser vistas en el Tabla 1, se observa una gran variabilidad en estos resultados, desde un mínimo de 74,4 cercarias en promedio para la cepa de Mariara y un máximo de 591,7 para la cepa de Chabasquén y 528,3 para del Estado Lara.

Para el mismo criterio producción de cercarias al tercer día de iniciada la emisión, el análisis de varianza de Kruskal-Wallis arrojó diferencias estadísticamente significativas entre las 9 cepas de $B$. glabrata y la prueba de la mínima diferencia significativa, entre los rangos de emisión de cercarias, reflejó la existencia de diferencias estadísticamente significativas entre casi todas las 36 combinaciones de cepas de B. glabrata, a excepción de las cuatro siguientes: Mariara/ Ingenio Bolívar, Cagua/Caserío El 25, Lago de Valencia/Güigüe y Güigüe/ Caripe (Tabla 2).

En la Tabla 3 se presenta un resumen de los criterios considerados en el presente trabajo, para evaluar la compatibilidad hospedador intermediario-parásito, interpretados cualitativamente. 
Tabla 2 - Análisis de varianza de Kruskal-Wallis y mínima diferencia significativa para la producción de cercarias al tercer día de iniciada la emisión en nueve cepas de B. glabrata experimentalmente infectadas con la cepa C5 de S. mansoni.

\begin{tabular}{lccccccccc}
\hline & C1 & C2 & C3 & C4 & C5 & C6 & C7 & C8 & C9 \\
\hline C1 & & 239,3 & 211,6 & 188,5 & 122,3 & 125,9 & 274,9 & 96,8 & 46,9 \\
C2 & S & & 450,9 & 50,8 & 361,6 & 365,3 & 514,3 & 142,5 & 192,4 \\
C3 & S & S & & 400,1 & 89,3 & 85,6 & 63,4 & 308,4 & 258,5 \\
C4 & S & N.S. & S & & 310,8 & 314,5 & 463,5 & 91,7 & 141,6 \\
C5 & S & S & S & S & & 3,7 & 152,7 & 219,1 & 169,2 \\
C6 & S & S & S & S & N.S. & & 148,9 & 222,8 & 172,9 \\
C7 & S & S & S & S & S & S & & 371,8 & 321,9 \\
C8 & S & S & S & S & S & S & S & S & N.S. \\
C9 & N.S. & S & S & S & S & S & S & \\
\hline
\end{tabular}

$\mathrm{H}=97,4(\mathrm{P}<0,05) \mathrm{G} . \mathrm{L} .=8$, m.d.s. $=56,5$

$\mathrm{H}$ = estadístico de la prueba de Kruskal-Wallis; G.L. = grados de libertad; m.d.s. = mínima diferencia significativa; $\mathrm{S}=$ diferencias estadísticamente significativas; N.S. = no hay diferencias estadísticamente significativas; $C 1$ = cepa de $B$. glabrata Lago de Valencia; C2 = cepa de Mariara; C3 = cepa de Anzoátegui (Edo. Lara); C4 = cepa de Ingenio Bolívar; C5 = cepa de Cagua; C6 = cepa de Caserío El 25; C7 = cepa de Chabasquén; C8 = cepa de Caripe; C9 = cepa de Güigüe.

Tabla 3 - Cuadro resumen de los criterios de compatibilidad considerados para las 9 cepas evaluadas de B. glabrata experimentalmente infectadas con la cepa C5 de S. mansoni.

\begin{tabular}{|c|c|c|c|c|}
\hline$\overline{\text { Cepa }}$ & Area & PPIM & $\overline{D I}$ & NCP \\
\hline & & & & $3^{\text {er }}$ día \\
\hline Lago de Valencia & end. & corto & corta & $A$ \\
\hline Cagua & end. & corto & corta & A \\
\hline Ingenio Bolívar & end. & corto & mod. & B \\
\hline Mariara & end. & corto & corta & B \\
\hline Caserío El 25 & end. & corto & larga & A \\
\hline Güigüe & end. & corto & larga & A \\
\hline Anzoátegui & no end. & corto & corta & A \\
\hline Chabasquén & no end. & corto & corta & A \\
\hline Caripe & no end. & corto & corta & A \\
\hline
\end{tabular}

end. = endémica; $\mathrm{PPIM}=$ período prepatente intramolusco; $\mathrm{DI}=$ duración de la infección; $\mathrm{NCP}=$ número de cercarias producidas al $3^{\text {er }}$ día de iniciada la emisión; $A=$ abundante; $B$ = bajo.

\section{DISCUSION}

Diversos autores han señalado la gran variabilidad existente en la compatibilidad entre las cepas de $S$. mansoni y B. glabrata en Sur América ${ }^{4}{ }^{12}$. Frandsen ${ }^{8}$ considera que dicha variabilidad es debida a la composición genética compleja del parásito, lo cual es resultado de la mezcla al azar de varias poblaciones de $S$. mansoni de Africa que fueron introducidos en América en épocas coloniales a través de los esclavos. Evidencias sobre esa variabilidad genética fue recientemente reportada por Minchella y cols ${ }^{10}$, quienes comprobaron en un estudio realizado en Belo Horizonte (Brasil) que mas de la mitad de los caracoles infectados en condiciones naturales albergaban diversos genotipos de S. mansoni.
La susceptibilidad de los moluscos hospedadores intermediarios a la infección por $S$. mansoni ha sido relacionada con la acción patógena del trematodo. En efecto, se ha señalado para el sistema S. mansoni - B. tenagophila (S.J., Brasil) que a mayor susceptibilidad, hubo mayor número de huevos en las heces, de granulomas hepáticos ${ }^{17}$, mayor mortalidad de los ratones infectados y menor peso corporal y de las visceras ${ }^{18}$.

Por otra parte, de Souza y cols ${ }^{15}$, refieren en hospedadores finales no humanos alteraciones en el sex ratio, en relación al grado de compatibilidad entre las cepas del sistema parásito - hospedador intermediario, en el sentido de que a mayor compatibilidad entre ellas, habrá mayor número de hembras de $S$. mansoni. 
En fin, puede considerarse que existe un polimorfismo de compatibilidad, que es expresión del polimorfismo genético de Schistosoma confrontado al del molusco lo cual aporta datos importantes para estudios de funcionamiento de focos y para la evaluación del riesgo de desplazamiento de las poblaciones humanas contaminadas ${ }^{7}$.

Entre los criterios señalados por los autores como indicadores de compatibilidad entre caracoles hospedadores intermediarios y parásitos ${ }^{78}$, tenemos la duración del período prepatente intramolusco, el cual mientras mas corto indica mayor compatibilidad ${ }^{7}{ }^{16}$. La duración del mencionado período es variable, así vemos como entre cepas simpátricas de $B$. glabrata y $S$. mansonicomo las de Belo Horizonte (Brasil) se ha señalado una duración de 30 a 35 días, mientras que para cepas de $S$. mansoni como la de Sao José dos Campos (Brasil) que utiliza a Biomphalaria tenagophila Orbigny, 1835 hospedador intermediario se han señalado de 30 a 70 días $^{13}$.

Para las cepas venezolanas de S. mansoni B. glabrata, Chrosciechowski ${ }^{6}$ ha reportado de 28 a 30 días de duración. Los resultados del presente trabajo tanto para caracoles procedentes del área endémica tradicional de transmisión de esquistosomiasis como de fuera de ella, fueron bastante homogéneos y mas cortos, de 23 a 25 días de duración.

En lo referente a otro criterio de compatibilidad, la duración de la infección en días, se observa que las cepas de $B$. glabrata ubicadas fuera del área endémica tradicional presentaron un período de producción cercariano corto, lo cual es indicativo de poca compatiblidad ${ }^{7}$, mientras que las procedentes del área bilharzígena tradicional fueron bastante variables.

Desde el punto de vista de la producción cercariana, la evaluación del total de dichas larvas obtenidas al tercer día de iniciada la emisión, permitió evidenciar que de las cepas de $B$. glabrata que produjeron mas de 10.000 cercarias, dos proceden de localidades ubicadas fuera del área endémica tradicional de transmisión de la esquistosomiasis mansoni (Chabasquén y Anzoátegui).

El hecho de haber detectado diferencias estadísticamente significativas para el número de cercarias producidas en el tercer día de iniciada la emisión para la casi totalidad de combinaciones de cepas de B. glabrata evaluadas, evidencia un patrón de comportamiento distinto, lo cual es reflejo de la variabilidad de la compatibilidad existente entre las cepas del caracol y la cepa del parásito. en condiciones naturales para la cepa BH (Minas Gerais, Brasil) de Souza y cols ${ }^{14}$ reportan una emisión promedio por caracol de 17442 cercarias. Mientras que para la misma cepa pero en condiciones de laboratorio se obtuvo una emisión de 6061 cercarias/caracol, es decir 28 veces menos que en condiciones naturales, lo cual puede ser debido a que la mencionada cepa ha sido mantenida durante muchos años en el laboratorio lo cual conlleva a la pérdida de la variabilidad genética y puede ir en detrimento de la cantidad de cercarias emitidas.

Al interpretar los criterios de compatibilidad evaluados en conjunto para las nueve cepas de $B$. glabrata consideradas, concluimos que para las zonas bilharzígenas tradicionales, las cepas que revelaron mayor compatibilidad fueron las de Caserío El 25 y Güigüe, que presentaron períodos prepatentes intramolusco cortos, duración de la infección larga, alto número de días de producción abundante de cercarias y elevada producción de cercarias al tercer día de iniciada la emisión. Mientras que para las áreas no bilharzígenas, las cepas evaluadas tuvieron un patrón de comportamiento bastante similar, reflejando poca compatibilidad, resultando ligeramente mas compatible la cepa de Anzoátegui (Edo. Lara).

Otro aspecto evaluado, la cura espontánea, el cual no ha sido incluido por los autores ${ }^{78}$ como indicador de compatibilidad, pero que se consideró interesante debido a las implicaciones epidemiológicas que puede tener una cepa que se negativice espontáneamente en elevado porcentaje y pueda estar en condiciones de volver a infectarse y mantener un foco esquistosomiásico activo. Entre las cepas evaluadas en el presente trabajo, el mas bajo porcentaje de autocuración se detectó en la cepa de Güigüe con $21,4 \%$, pero se obtuvieron porcentajes bastante elevados de mas del $80 \%$ en las cepas del Lago de Valencia (Aragua), Anzoátegui (Lara), Cagua (Aragua), Chabasquén (Portuguesa) y Caripe (Monagas). Como se observa tres de las cinco cepas mencionadas con los mas elevados porcentajes de autocuración están ubicadas fuera del área endémica tradicional de transmisión lo cual les confiere importancia epidemiológica. Sería importante evaluar estas cepas en condiciones naturales, en vista de que en el Brasil de Souza y cols ${ }^{14}$ refieren la presencia 
de autocuración en un $13 \%$ de los caracoles infectados en condiciones naturales.

A manera de conclusión podemos decir que de estudios como el presente se deriva información importante sobre el comportamiento de las cepas venezolanas en condiciones de laboratorio, ya que de acuerdo a la finalidad que se tenga, podríamos escoger la cepa de $B$. glabrata o el sistema B. glabrata- S. mansonimas adecuado. Por ejemplo, en estudios de bioquímica o inmunología en los cuales se requiere en corto tiempo hasta un millón de cercarias frescas ${ }^{9}$, se podrían escoger las cepas mas rendidoras como las de Chabasquén, Cagua, Caserío El 25 o Anzoátegui que emitieron mas de 10000 cercarias al tercer día de iniciada la emisión. Mientras que si se requiere mantener la producción de cercarias por períodos mas prolongados, se podrían escoger cepas como Güigüe o Caserío El 25 que presentaron 93 y 64 días de emisión respectivamente.

\section{REFERENCIAS BIBLIOGRAFICAS}

1. Alarcón de Noya B, Noya O, Balzán C, Césari I. New approaches for the control and erradication of schistosomiasis in Venezuela. Memorias do Instituto Oswaldo Cruz 87:227-231, 1992.

2. Alarcón de Noya B, Césari Y, Losada S, Colmenares C, Balzán C, Hoebeke J, Noya O. Evaluation of alkaline phosphatasa immunoassay and comparison with other diagnostic methods in areas of low transmission of schistosomiasis. Acta Tropica 66:69-78, 1997.

3. Barbosa F. Proven and potencial vectors of the trematode Schistosoma mansoni in South America. Revista Brasilera de Biologia 20:183-190, 1960.

4. Barbosa S, Barreto C. Differences in susceptibility of brazilian strains of Australorbis glabratus to Schistosoma mansoni. Experimental Parasitology 9:137-140, 1960.

5. Barbosa F, Coelho M. Açâo da dessecaçâo sôbre as fases larvarias intracaramujo de Schistosoma mansoni em Australorbis glabratus. Publicaçoes Avulsas do Instituto de Pesquisas Aggeu Magalhâes 2:159-162, 1953.

6. Chrosciechowski P. El caracol Biomphalaria glabrata en Venezuela. (Mimeografiado), M.S.A.S., Maracay, Venezuela, 1988.

7. Combes C. L'analyse de la compatibilité Schistosomes/ mollusques vecteurs. Bulletin de la Societé de Pathologie Exotique 78:742-746, 1985.

8. Frandsen, F. Discussion of the relationships between Schistosoma and their intermediate hosts, assessment of the degree of host parasite compatibility and evaluation of Schistosoma taxonomy. Parasitenkunde 58:275-296, 1979.

9. Lewis F, Stirewalt M, Souza C, Gazzinelli G. Large - scale laboratory maintenance of Schistosoma mansoni, with observations on three Schistosoma/snail host combinations. Journal of Parasitology 72:813-829, 1986.

10. Minchella D, Sollenberger K, Pereira de Souza C. Distribution of Schistosoma genetic diversity within molluscan intermediate hosts. Parasitology 111:217-220, 1995.

11. Morales G, Pino LA. Parasitometría. Ediciones Universidad de Carabobo, Valencia, 1995.

12. Paraense W, Correa L. Variations in susceptibility of population of Australorbis glabratus to strain of Schistosoma mansoni. Reevista do Instituto de Medicina tropical de Sao Paulo 5:15-22, 1963.

13. Paraense W, Correa L. Observations on two biological races of Schistosoma mansoni. Memorias do Instituto Oswaldo Cruz 76:287-291, 1981.

14. Souza C, Araujo N, Jannotti - Passos L, Guimares C. Production of Schistosoma mansoni cercariae by Biomphalaria glabrata from a focus in Belo Horizonte, Minas Gerais. Revista do Instituto de Medicina Tropical de Sao Paulo 36:485-489, 1994.

15. Souza C, Jannotti-Passos S, Ferreira S, Vieira I. Schistosoma mansoni: the sex ratios of worms in animalsinfected with cercariae from three species of Biomphalaria. Revista do Instituto de Medicina Tropical de Sao Paulo 38:141-146, 1996.

16. Southgate V, Rollinson D, Ross G, Knowless R. Observations on an isolate of Schistosoma bovis Tanzania. Zoological Parasitenkunde 63:241-249, 1980.

17. Zanotty-Magalhaes E, Magalhaes L, Carvalho J. Relaçao entre a patogenicidade do Schistosoma mansoni em camundongos e a susceptibilidade do molusco vetor. II. Numero de ovos nas fezes e numero e tamanho dos granulomas nas visceras. Revista de Saude Publica 6:412-419, 1993.

18. Zanotti-Magalhaes E, Magalhaes L, Carvalho J. Relaçao entre a patogenicidade do Schistosoma mansoni em camundongo e a susceptibilidade do molusco vetor. III. Mortalidade, pesos corporal das visceras. Revista de Saúde Pública 29:265-270, 1995. 